\title{
RENTABILIDAD DE SORGO (Sorghum bicolor L. MOENCH) EN EL MUNICIPIO DE SAN FERNANDO, TAMAULIPAS ${ }^{1}$
}

\author{
José Luis Machuca ${ }^{2}$, Raúl Rodríguez ${ }^{3}$,Héctor Williams ${ }^{3}$, Noé Montes ${ }^{3}$, Jesús Loera ${ }^{3}$
}

\section{RESUMEN}

Rentabilidad de sorgo (Sorghum bicolor L. Moench) en el municipio de San Fernando, Tamaulipas. En el municipio de San Fernando, Tamaulipas, México se analizaron los costos y beneficios que obtuvieron los agricultores con el sorgo, para determinar por qué se sigue sembrando esta poasea. Se estimaron los costos de producción del rancho "El Paraíso" y del ejido "El Casanaleño" durante el período 1987-91 y mediante encuestas se determinó el costo promedio de producción del cultivo en la zona. Con base en los rendimientos y los precios nacional e internacional a tasas de interés de los créditos bancarios, se estimó el indicador de rentabilidad (Valor Actual Neto, Tasa Interna de Retorno y la Relación Beneficio-Costo) para el ciclo agrícola otoño-invierno 1990-91. Para el promedio de los agricultores, los costos de producción fueron $57 \%$ menores que los programados por la banca comercial, dado que no aplican fertilizantes e insecticidas y realizan menor número de labores agrícolas. Esto significa que de acuerdo a los niveles de producción actual, los precios de venta en México y costos de producción reales, este cultivo resultó redituable. Sin embargo no fue redituable, al considerar el precio internacional sin arancel.

\begin{abstract}
Sorghum (Sorghum bicolor L. Moench) profitability in the San Fernando, Tamaulipas District. Sorghum profitability was studied in San Fernando, Tamaulipas, in arder to determine the reasons for which farmers of this region continue to grow the crop. Production costs of Rancho "El Paraíso" and of the "El Casanaleño" commons during the period 1987-91 were estimated, and a survey was carried out in the same period to determine the average cost of sorghum production in the area. The profitability indicator (current net value, return rate, and cost-efficiency ratio) for the fa11winter 1990-91 agricultural period was estimated based on yield, national and international prices, and interest rates of crediting banks. Results indicated that for regular farmers production costs were $57 \%$ lower than those estimated by commercial banks, because farmers do not use fertilizers or pesticides, and they undertake fewer agricultural tasks. Taking into account current production levels, sale prices in Mexico, and actual production costs, this crop turns out to be profitable. However, if international duty-free prices are considered, sorghum production is not profitable.
\end{abstract}

\section{INTRODUCCIÓN}

En el Municipio de San Fernando Tamaulipas se siembran alrededor de 276 mil hectáreas en época lluviosa, de éstas el $96 \%$ se dedica al cultivo del sorgo. En esta superficie se producen 473 mil toneladas de grano, (nueve porciento del total de la producción nacional de este cereal). Las condiciones de precipitación en el municipio son erráticas, sin embargo, los rendimientos promedios durante el período de 1988-92 se han mantenido en dos toneladas por hectárea. A pesar de estas condiciones climáticas, el índice de pérdidas representa sólo el 5,2\% SARH (1992). En los últimos años, los productores de sorgo de esta zona han manifestado problemas de insolvencia financiera por la reducción del precio del grano y el incremento en los costos de producción.

Durante el período de 1989-91 los Fideicomisos Instituidos en Relación con la Agricultura (FIRA), se vieron en la necesidad de reestructurar el 16,39\% de los créditos otorgados. El anterior porcentaje se ha considerado superior al ocho por ciento de cartera vencida, que los funcionarios de la banca establecieron como límite en la actividad agropecuaria. Otra consecuencia de dicha insolvencia financiera fue la disminución en 39\%

1 Presentado en la XLIII Reunión anual del PCCMCA en Panamá 1997.

2 Parte de la tesis del primer autor. Universidad Autónoma Chapingo, Chapingo, México. 56230.

3 Campo Exp Río Bravo-INIFAP. Investigador del Programa de Cultivos Industriales. Campo Experimental Río Bravo-INIFAP. Ap. Postal 172. Río Bravo, Tam. 88900. 
de la superficie habilitada por la banca comercial durante el período de 1988-1992 (FIRA, 1992). Como una respuesta a esta situación, la Secretaría de Agricultura y Recursos Hidráulicos (SARH), ha planteado la necesidad de modificar el sistema de monocultivo de la región, o bien introducir en ésta otro cultivo más redituable. Sin embargo, por ser ésta una región semidesértica, de clima extremoso y suelos pobres, son pocos los cultivos que pueden utilizarse como alternativa. Uno de los cultivos con mayor potencial desde el aspecto de manejo y comercialización, es el trigo, el cual a pesar de los esfuerzos por introducirlo a la región, no ha despertado interés entre los agricultores, ya que sólo e14\% de la superficie se dedica a esta graminea (SARH, 1992). Esto ha hecho necesario analizar de manera más objetiva los costos y beneficios que obtienen los agricultores con el sorgo para poder determinar el por qué se sigue sembrando, a pesar de tener aparentemente en su contra altos costos de producción y bajos precios del grano.

Los cultivos de sorgo y el maíz surgieron como una alternativa al cultivo del algodón en el norte de Tamaulipas (Talavera; Guerra 1962). La Secretaría de Agricultura y Recursos Hidráulicos (1984) indica que en esta región el cultivo del sorgo fue rápidamente adoptado, ejemplo de ello es que en 1956 se sembraron 3.700 hectáreas, mientras que para 1983 la superficie sembrada de sorgo bajo riego fue de 122 mil hectáreas y 535 mil hectáreas de temporal. En el municipio de San Fernando, se sembraron en 1961 casi tres mil hectáreas de sorgo (Talavera; Guerra 1962). Esta superficie, después de 30 años se incrementó en un siete porciento llegándose a sembrar en promedio 224 mil hectáreas durante el período de 1988-92.

La superficie promedio sembrada de sorgo en México durante el período de 1980-90, fue de casi dos millones de hectáreas. Esto lo ubica como el tercer cultivo en importancia después del maíz y frijol (Salcedo, 1991). La producción de sorgo en el mismo período ha t1uctuado en un promedio de 5,3 millones de toneladas, mostrando una tasa de crecimiento del $0,4 \%$. Este volumen de producción ubica al sorgo como el segundo cultivo en importancia después del maíz. En relación a esto, Zavala (1984) señala que a pesar de la importancia de este cultivo y de la gran superficie sembrada, la producción no ha sido suficiente para abastecer al mercado nacional al existir un déficit de aproximadamente dos millones de toneladas anuales. Los precios reales del sorgo, tanto en el ámbito nacional como internacional han tenido un comportamiento a la baja durante el período de 1980-90 (Salcedo, 1991). De 1980 a 1987 los precios internacionales tuvieron una disminución del $43,2 \%$ y entre $1987-90$ el deterioro fue del $18,9 \%$,con respecto a 1980. En México los precios en 1984 tuvieron un incremento en términos reales del $17,8 \%$, pero en 1988 y 1990 tuvieron una disminución mayor que los precios internacionales, lo cual ha acarreado inconformidad por parte de los productores, debido a que en el mismo período los costos se continuaron elevando, el mismo autor menciona que las causas de la baja del precio del sorgo en México, son diferentes a las de Estados Unidos. En este caso, se debió a un esfuerzo del Gobierno Mexicano por abatir la inflación.

\section{MATERIALES Y MÉTODOS}

El presente trabajo se realizó en el municipio de San Fernando, ubicado en la parte norte del estado de Tamaulipas, latitud norte $24^{\circ} 50^{\prime}$ y $25^{\circ} 40^{\prime}$ y longitud oeste $98^{\circ} 09^{\prime}$ y $97^{\circ} 20^{\prime}$. El análisis se efectuó con la información obtenida durante el ciclo agrícola otoño-invierno 1990-91, debido a que fue el más representativo del período comprendido entre 1987 y 1992.

Los costos de producción se determinaron con base en los registros contables que se llevaron a cabo en el rancho "El Paraíso", propiedad del Sr. José Q. Medina Galván, ubicado en el ejido Lavaderos, en el período contable de 1987-88 y en el ejido "El Casanaleño" ubicado en San Fernando en el período contable de 1988-91. Por otra parte, con la finalidad de tener una perspectiva más amplia de la tecnología usada en la producción de sorgo de temporal, se entrevistaron 10 pequeños propietarios y 20 ejidatarios. La distribución de los productores encuestados fue de acuerdo a las áreas más representativas de la región, siendo estas: Santa Teresa, La Laguna y la cabecera municipal.

Las labores de preparación y cultivo para determinar el costo de producción, se basaron en la tecnología usada por un agricultor promedio. Para la asignación del costo de cada labor, se utilizaron los establecidos con fines de crédito por FIRA.

Se cuantificó como rendimiento promedio de grano (t/ha) a la media de producción del período 1988-92 y como precio de venta $\$ 370,00$ el cual fue el precio de garantía establecido para el ciclo otoño-invierno 199091; así también, el precio internacional de $\$ 84,49$ que fue el precio establecido para el mes de julio de 1991. Los beneficios obtenidos se estimaron multiplicando la producción por hectárea, por el precio de garantía en México y por el precio internacional. La Tasa de Interés fue estimada en $2,75 \%$, tomando como base el costo de oportunidad del dinero, definido por el Costo Porcentual Promedio (C.P.P.) fijado por el Banco de México para el mes de julio, fecha en que se iniciaron 
las labores de preparación del terreno. Se determinaron los costos y beneficios, se procedió al cálculo de los indicadores de rentabilidad (Valor Actual Neto, Tasa Interna de Retorno y Relación Beneficio-Costo) de acuerdo a las fórmulas propuestas por Gittinger (1973), calculados tanto éon el precio de garantía en México, como con el precio internacional. Para la estimación de estos indicadores, se tomó en cuenta la tecnología más usada por los agricultores en la producción de sorgo de temporal.

\section{RESULTADOS Y DISCUSIÓN}

En el rancho "El Paraíso", los costos de producción del sorgo se distribuyeron de la siguiente manera: $22,4 \%$ en maquinaria, $13,62 \%$ en in sumos, $7,22 \%$ en mano de obra, $53,84 \%$ en trilla y flete, y en otros gastos el 2,92\% (Cuadro 1). Mientras que en el ejido "El Casanaleño" los costos de producción por hectárea fueron: $28,42 \%$ en maquinaria; $24,05 \%$ en mano de obra; $15,23 \%$ en insumos; $25,47 \%$ en trilla y acarreo, y $6,83 \%$ en otros gastos. En estas explotaciones, los costos de producción por hectárea durante los ciclos agrícolas $87-88,88-89,89-90$ y $90-91$, representaron el $44,07 \% ; 51,36 \% ; 39,30 \%$ y $26,28 \%$; respectivamente del costo del cultivo que PIRA programó con fines de crédito (Cuadro 2).

Los gastos en maquinaria fueron similares en el rancho "El Paraíso" y en el ejido "El Casanaleño" lo que muestra que éstos son los costos promedio reales que puede tener un agricultor en la región. En cuanto a la mano de obra se encontró una diferencia de 16,8\% entre explotaciones agrícolas, debido a que en el rancho
"El Paraíso" el costo incluyó un trabajador de planta, el operador de maquinaria y los pagos de las deshierbas. Por esta razón, se puede considerar la explotación de este rancho más eficiente que la del ejido.

El costo de los insumos fue similar para ambas explotaciones, ya que éste se limitó a la compra de semilla para la siembra. En la región, generalmente el agricultor evitó el uso de insecticidas al sembrar en las fechas recomendadas por la SARH y el control de malezas lo efectuó en forma mecánica y manual; la aplicación de fertilizantes fue mínima. Al respecto, Salceda (1991) señala que en los principales estados productores de sorgo se acostumbra aplicar fertilizantes, excepto en Tamaulipas, donde la fertilización es mínima.

El costo de la trilla y acarreo en el rancho "El Paraíso" representó $53,8 \%$ del costo total del cultivo (Cuadro 1), dado que tiene vías de acceso difíciles y alejadas que hicieron que esta labor se incrementara hasta el doble de lo que se paga normalmente. En el ejido el costo de trilla y acarreo significó el $25,42 \%$ y correspondió al porcentaje promedio que hace el agricultor. En igualdad de condiciones el rancho "El Paraíso" incrementaría su rentabilidad. En el ciclo agrícola 1988-89, el costo del cultivo fue alto en comparación con los otros ciclos agrícolas, debido al pago del seguro agrícola.

La información obtenida de los agricultores encuestados en las tres áreas estudiadas del municipio de San Fernando, indicó que en promedio el $85 \%$ de los agricultores basan su preparación en rastreas y bordeos. La poca aceptación del barbecho se debe a su alto

Cuadro 1. Costos reales (dólares) de producción de sorgo de temporal en dos localidades del municipio de San Fernando, Tamaulipas, México. 1987-1991.

\begin{tabular}{|c|c|c|c|c|c|c|}
\hline \multicolumn{7}{|c|}{ Ciclos Agrícolas } \\
\hline \multicolumn{3}{|c|}{ Rancho El Paraíso } & \multicolumn{4}{|c|}{ Ejido El Casanaleño } \\
\hline Concepto & 1987-88 & $\%$ & 1988-89 & $1989-90$ & $1990-91$ & $\%$ \\
\hline Maquinaria & $6.305,00$ & 22,40 & 15.710 & $13.809,00$ & $22.280,00$ & 28,42 \\
\hline Mano de obra & $2.029,00$ & 7,22 & 10.356 & $16.823,00$ & $17.085,00$ & 24,05 \\
\hline Insumos & $3.831,00$ & 13,62 & 9.716 & $11.560,00$ & $5.955,00$ & 15,23 \\
\hline Seguro Agrícola & - & & 29.376 & - & - & \\
\hline Trilla & $15.139,00$ & 53,84 & 18.812 & $20.672,00$ & $12.834,00$ & 25,47 \\
\hline Otros & 816,00 & 2,92 & 4.692 & - & $7.682,00$ & 6,43 \\
\hline Total & $28.120,00$ & 100,00 & 82.662 & $62.864,00$ & $65.836,00$ & 100,00 \\
\hline ha & 200,00 & & 272 & 272,00 & 272,00 & \\
\hline Costo/ha & 140,60 & & 303,90 & 231,11 & 242,04 & \\
\hline
\end{tabular}

Nota: Maquinaria incluye: Combustible, lubricantes y reparaciones de la maquinaria agrícola y del vehículo. Insumos: semilla. Mano de obra: control de malezas. 
Cuadro 2. Comparación de los costos de producción por hectárea, programados (C.P.) y reales (C.R.) del sorgo de temporal en dos localidades de San Fernando, Tamaulipas, México. 1987-1991.

\begin{tabular}{|c|c|c|c|c|c|c|c|c|}
\hline \multirow[b]{4}{*}{ Concepto } & \multirow{3}{*}{\multicolumn{2}{|c|}{$\begin{array}{c}\text { Rancho El Paraíso } \\
\text { Ciclo Agrícola } \\
1987-1988\end{array}$}} & \multirow{2}{*}{\multicolumn{6}{|c|}{ Ejido El Casanaleño }} \\
\hline & & & \multicolumn{4}{|c|}{ Ciclo Agrícola } & & \\
\hline & & & \multicolumn{2}{|c|}{ 1988-1989 } & \multicolumn{2}{|c|}{$1989-1990$} & \multicolumn{2}{|c|}{ 1990-1991 } \\
\hline & C.P. & C.R. & C.P. & C.R. & C.P. & C.R. & C.P. & C.R. \\
\hline Maquinaria & 100,8 & 31,5 & 246,4 & 57,7 & 227,0 & 77,9 & 232,0 & 106,5 \\
\hline Mano de obra & 19,6 & 10,2 & 22,5 & 32,1 & 30,0 & 34,7 & 30,0 & 38,2 \\
\hline Insumos & 67,4 & 19,2 & 93,7 & 35,7 & 93,0 & 42,5 & 107,0 & 21,9 \\
\hline Seguro & 49,4 & - & 108,0 & 108,0 & 108,0 & - & 112,0 & - \\
\hline Cosecha & 56,8 & 75,7 & 81,0 & 47,1 & 78,0 & 76,0 & 100,0 & 47,2 \\
\hline Otros & 25,0 & 4,1 & 40,0 & 17,3 & 52,0 & - & 40,0 & 28,3 \\
\hline Total & 319,0 & 140,6 & 591,6 & 303,9 & 588,0 & 231,1 & 621,0 & 242,1 \\
\hline \% C.R. vs C.P. & & 44,07 & & 51,36 & & 39,30 & & 26,28 \\
\hline
\end{tabular}

costo aunado al riesgo e incertidumbre que se tiene sobre la presencia de lluvias suficientes para obtener una producción aceptable (Cuadro 3).

Los costos programados con fines de crédito, se elaboraron tomando en cuenta que el productor realizó las labores de acuerdo al paquete tecnológico recomendado para el cultivo del sorgo; sin embargo, se observó que las labores de preparación de los terrenos se limitaron a rastreos y bordeos, razón por la que al momento de contabilizar los costos de producción realizados por el productor, estos fueron más bajos en comparación con los programados. De acuerdo a esto, Sánchez (1980) mencionó que el sorgo fue rentable, dado que muchos agricultores lo cultivaron con una tecnología parecida a la labranza mínima disminuyendo el costo del cultivo.

El costo de producción realizado para fines de estudio se obtuvo de las encuestas efectuadas y se le agre-

Cuadro 3. Labores culturales y porcentaje de productores que realizan estas prácticas en tres áreas de estudio.

\begin{tabular}{|c|c|c|c|c|c|c|}
\hline \multirow[t]{2}{*}{$\begin{array}{l}\text { Labores } \\
\text { culturales }\end{array}$} & \multicolumn{2}{|c|}{$\begin{array}{l}\text { Santa } \\
\text { Teresa }\end{array}$} & \multicolumn{2}{|c|}{$\begin{array}{c}\text { La } \\
\text { Laguna }\end{array}$} & \multicolumn{2}{|c|}{$\begin{array}{c}\text { C. } \\
\text { Municipal }\end{array}$} \\
\hline & P.P.1 & E. 2 & P.P. & E. & P.P. & E. \\
\hline Barbecho & 33 & 26.6 & 0 & 0 & 10 & 0 \\
\hline Rastreo & 100 & 100 & 100 & 100 & 100 & 100 \\
\hline Bordeo & 100 & 100 & 100 & 100 & 100 & 100 \\
\hline Déshierba & 100 & 100 & 100 & 100 & 100 & 100 \\
\hline
\end{tabular}

$1=$ Pequeños propietarios. $\quad 2=$ Ejidatarios. gó como un dato adicional, el valor de la renta de la tierra. El costo de cada labor se programó basándose en el costo que PIRA aseguró para el ciclo otoño-invierno 1990-91 (Cuadro 4).

En relación a los precios internacionales el sorgo también ha tenido una disminución en términos reales que afectan totalmente la rentabilidad del cultivo. El precio internacional para el mes de julio de 1991 fue de $\$ 84,49$, el cual a una cotización de \$ 3.334, más los gastos de importación representó \$263,80, sin tomar en cuenta el arancel.

El precio internacional del sorgo es precisamente uno de los principales factores que determinan las ventajas competitivas de la producción del sorgo en México y que la variabilidad del precio internacional del sorgo constituye uno de los principales problemas para los productores en un acuerdo de libre comercio. Sin embargo, los precios del sorgo concertados por el Gobierno Federal, a través del organismo de Apoyos y Servicios a la Comercialización Agropecuaria (ASER$\mathrm{CA}$ ), han hecho que el precio del sorgo se mantenga por arriba del precio internacional y que haga rentable dicha actividad.

Según los resultados, los beneficios brutos obtenidos con el precio nacional del sorgo fueron de $\$ 740,00$; mientras que con el precio internacional fueron de \$527,60 (Cuadro 5).

El período de cultivo del sorgo es de un año y como el valor del dinero cambia a través del tiempo, es indispensable utilizar un procedimiento que permita comparar capacidades de pago y compromisos de créditos con pesos de la misma fecha. El método normal de 
Cuadro 4. Costos unitarios $(\$)$ de labores agrícolas del cultivo de sorgo de temporal durante el ciclo agrícola otoño-invierno 1987-91.

\begin{tabular}{|c|c|c|c|c|c|c|c|c|c|c|c|c|}
\hline Concepto & Tot & jul & ago & sep & oct & nov & dic & ene & feb & mar & abr may & jun \\
\hline \multicolumn{13}{|l|}{ Preparación terreno } \\
\hline Labores de terreno & 28 & 28 & & & & & & & & & & \\
\hline Bordeo & 25 & & 25 & & & & & & & & & \\
\hline Contrabordeo & 25 & & & 25 & & & & & & & & \\
\hline Rastreo & 28 & & & & & 28 & & & & & & \\
\hline Rastreo presiembra & 28 & & & & & & & 28 & & & & \\
\hline \multicolumn{13}{|l|}{ Siembra } \\
\hline Semilla & 28 & & & & & & & & 28 & & & \\
\hline Siembra & 49 & & & & & & & & 49 & & & \\
\hline \multicolumn{13}{|l|}{ Labores culturales } \\
\hline Cultivo & 24 & & & & & & & & & 24 & & \\
\hline Cultivo & 24 & & & & & & & & & & 24 & \\
\hline Deshierbe & 30 & & & & & & & & & & 30 & \\
\hline Insecticida & 23 & & & & & & & & & & 23 & \\
\hline Aplicación & 15 & & & & & & & & & & 15 & \\
\hline Cosecha & 50 & & & & & & & & & & & \\
\hline Trilla & 40 & & & & & & & & & & & 50 \\
\hline Flete & & & & & & & & & & & & 40 \\
\hline Arrendamiento terreno & 185 & & & & & & & & & & & \\
\hline Renta & & & & & & & & & & & & 185 \\
\hline Total & 602 & 28 & 25 & 25 & & 28 & & 28 & 77 & 24 & 92 & 275 \\
\hline
\end{tabular}

Cuadro 5. Beneficios brutos (\$/ha) estimados para el cultivo del sorgo de temporal durante el ciclo otoño-invierno 1987-91.

\begin{tabular}{|c|c|c|c|}
\hline \multirow{2}{*}{$\begin{array}{l}\text { Rendimiento } \\
\text { (t/ha) }\end{array}$} & \multicolumn{2}{|c|}{ Precio del grano \$/t } & \multirow{2}{*}{$\begin{array}{r}\text { Beneficios } \\
\text { brutos } \$ / \text { ha }\end{array}$} \\
\hline & México & Internal. & \\
\hline 2,0 & 370,00 & & 740,00 \\
\hline 2,0 & & 263,80 & 527,60 \\
\hline
\end{tabular}

comparación es mediante el uso de la actualización y para esto los indicadores utilizados fueron: El Valor Actual Neto (VAN), la Tasa Interna de Retorno (TIR) y ]a relación Beneficio-Costo $(\mathrm{B} / \mathrm{C})$.

Con el precio de sorgo en México los indicadores económicos fueron un VAN positivo igual a 88,08; TIR $=7,06 \%$ y B $/ \mathrm{C}=1,17 ;$ al respecto, Gittinger $(1973)$ mencionó que el criterio de selección formal para ]a medida del VAN del proyecto consiste en aceptar todos los proyectos cuyo VAN sea positivo, a] actualizarlo al costo de oportunidad del capital. En relación a la TIR, el mismo autor menciona que se deben de aceptar todos aquellos proyectos en los cuales la tasa de rentabilidad interna sea superior al costo de oportunidad del capital; en este caso la TIR fue 4,31\% arriba de la tasa de interés para el mes de julio de 1990 (2,75\%).

Por otra parte, cuando se utiliza la relación beneficio-costo para evaluar proyectos, el criterio consiste en aceptar todos aquellos cuya relación beneficio-costo sea igual a uno o superior a uno. En este caso la relación $\mathrm{B} / \mathrm{C}$ indica que por cada peso que se invierte, se tiene una ganancia de 17 centavos, lo cual demuestra que el cultivo de sorgo es rentable.

Lo anterior coincide con López (1990), quien concluyó que el cultivo del sorgo de temporal es rentable en el ejido de Tlapeyecac, municipio de Cuautla, Morelos, ya que se obtuvo una ganancia total actualizada neta de $\$ 523,70$ por ha; por cada peso invertido se ganan 33 centavos; el rendimiento total del proyecto es de $7,49 \%$ el cual fue superior al costo de oportunidad real del capital $(1,08 \%)$ y los beneficios totales capitalizables son de \$578,60. Así mismo, Sánchez (1980), consignó que un estudio realizado en el sudeste del estado de Morelos, el cultivo del sorgo fue rentable pues el VAN dio mayor que cero, la TIR estuvo a casi ocho puntos porcentuales por arriba de la tasa de interés prevaleciente y la Relación B/C fue mayor que uno en casi 39 centavos. 
Con el precio internacional del sorgo los tres indicadores económicos $(\mathrm{VAN}=-84,65 ; \mathrm{TIR}=-6,85 \%$ y $\mathrm{B} / \mathrm{C}=0,87)$ muestran que bajo estas condiciones el cultivo no fue rentable, pues el VAN es menor que cero, la TIR se encuentra abajo de la tasa de interés para el mes de julio de 1990 (2,75\%) y la relación B/C nos indica que por cada peso invertido tenemos una pérdida de 13 centavos. Esto es sin considerar el arancel del 15\% que actualmente se aplica a la importación del sorgo. En caso de que se tome en cuenta este arancel los indicadores económicos resultantes serían $\mathrm{VAN}=-21,52$; $\mathrm{TIR}=-$ $3,28 \%$ y $\mathrm{B} / \mathrm{C}=0,99$. Esto demuestra también que bajo estas condiciones el sorgo no sería rentable. Para hacer que el cultivo sea negocio para los agricultores se tendría que aumentar el arancel del $15 \%$ al $28 \%$. Otra alternativa sería que el gobierno compensara el déficit que existe entre el precio que debe tener el sorgo nacional para hacerla rentable, aplicando subsidios similares a los que se aplican en los Estados Unidos de América. Salceda (1992) indicó que los subsidios que Estados Unidos ha otorgado a la producción del sorgo medido por los "Los Subsidios Equivalentes al productor" (SEP.) han variado de 16 a 55\%. Los datos más recientes se refieren a 1989, año en que los SEP se ubicaron en 33\%; en el caso de Tamaulipas para el área de temporal los SEP fueron de 12,2\%. Los subsidios equivalentes al productor cuantifican todas las transferencias directas e indirectas que recibe el productor a través de los distintos instrumentos de políticas, expresados en porcentajes que indican qué proporción del ingreso del productor son subsidiados.

Las condiciones climatológicas de la región, han permitido que el cultivo del sorgo se siga sembrando, pues bajo la tecnología de producción y los costos tan bajos sin incluir la renta de la tierra, se ha mantenido un promedio de dos toneladas por hectárea, que de alguna manera hace que el productor viva de esta actividad agrícola.

Con los costos reales obtenidos en las dos explotaciones a través de los años de estudio se requirió de 622; 949; 641 y 654 kg/ha para pagar la inversión. Si esto lo comparamos con la producción promedio regional se encuentra por abajo de los rendimientos prome dios; debido a esto el productor está consiente de que el sorgo es redituable de acuerdo al precio nacional.
En el municipio de San Fernando, Tamaulipas, a nivel medio el agricultor que siembra sorgo tuvo costos de producción $57 \%$ menor a los programados por la banca comercial, debido a que aplica un menor número de labores agrícolas y no utiliza fertilizantes e insecticidas. Bajo esas condiciones considerando los costos reales de producción y los precios del producto en México, el cultivo de sorgo es redituable. Se requirió un promedio $716 \mathrm{~kg} / \mathrm{ha}$ de grano para cubrir la inversión. Cuando se consideró el precio internacional con y sin arancel, el cultivo de sorgo no resultó redituable.

\section{LITERATURA CITADA}

GITIINGER, J.P. 1973. Análisis económicos de proyectos agrícolas. Editorial Tecnos. Madrid. España. pp. 12-119.

LÓPEZ, L.R. 1990. Rentabilidad del cultivo del sorgo (Sorghum bicolor L. Moench) en el ejido de Tlapeyecac, municipio de Cuautla, Morelos. Universidad Autónoma de Chapingo, México.

SALCEDO, B.S. 1991. Competitividad y ventajas comparativas del sorgo en México. Asociación Agrícola de Matamoros, Tamaulipas, México. p. 95.

SÁNCHEZ, D.D. 1980. Rentabilidad de los cultivos de maíz (Zea mays) Sorgo (Sorghum bicolor L. Moench) y Cacahuate (Arachis hipogaea L.) en el sudeste del Estado de Morelos. Tésis Universidad Autónoma de Chapingo. México. pp. 1-72.

SECRETARÍA DE AGRICULTURA Y RECURSOS HIDRAULICOS (SARH). 1984. Datos estadísticos de la región agrícola norte de Tamaulipas y Comité Mixto de promoción económica de Matamoros. 64 p.

SECRETARÍA DE AGRICULTURA Y RECURSOS HIDRAULICOS (SARH). 1992. Manual de cultivos del norte del Estado de Tamaulipas. Gobierno del Estado de Tamaulipas. México. pp. 54-63.

TALAVERA, E; GUERRA, O. 1962. Agricultura y ganadería en la región de Matamoros, Tam. Sociedad Agrónoma Mexicana sección Noreste de Tamaulipas. pp. 38-45.

ZAVALA, G.F. 1984. Estudio sobre el crecimiento y desarrollo del sorgo en México. Folleto de divulgación No.7. Universidad Autónoma de Nuevo León. México. pp. 4-11. 